\title{
Use of an asymmetric pulse profile for higher crystalline volumes from excimer laser crystallization of amorphous silicon
}

\author{
A. A. D. T. Adikaari, ${ }^{a)}$ N. K. Mudugamuwa, and S. R. P. Silva \\ Nano Electronics Centre, Advanced Technology Institute, University of Surrey, Guildford, \\ Surrey GU2 7XH, United Kingdom
}

(Received 19 February 2007; accepted 26 March 2007; published online 24 April 2007)

\begin{abstract}
An excimer laser is used to crystallize amorphous silicon on glass to nanocrystalline silicon, yielding higher crystalline volumes than reported earlier, by modifying the laser pulse profile used for crystallization at a given energy density. An asymmetric, shorter pulse profile, as opposed to the conventional Gaussian profile retains the desirable gradual leading edge of the Gaussian pulse for controlled evolution of hydrogen, while increasing the peak energy. The resultant films show an increased surface roughness along with higher crystalline volumes, which may be beneficial for photovoltaics and electron field emission cold cathodes. (C) 2007 American Institute of Physics.
\end{abstract}

[DOI: $10.1063 / 1.2731664]$

Excimer lasers have been utilized for the crystallization of hydrogenated amorphous silicon $(a-\mathrm{Si}: \mathrm{H})$ for electronic applications $^{1,2}$ with well established crystallization physics. ${ }^{3-7}$ Excimer lasers typically operate in the ultraviolet and hence photons are absorbed by the silicon thin films within a few nanometers of the surface. Melting and solidifying occur on a nanosecond time scale, often without affecting the underlying substrate. This technique enables the use of inexpensive substrates, such as glass, which are highly preferable for low cost, large-area electronic devices. Films with adequate thickness for light harvesting crystallize in the partial melting regime. ${ }^{2,3}$ The properties of crystallized silicon depend on a number of factors, with laser pulse shape being one of the most significant. Conventionally, a Gaussian profile along the long axis of the pulse has been reported to be best suited for controlled evolution of hydrogen during crystallization. ${ }^{8,9}$ Our previous report shows typical energy densities for crystallization with the crystalline volume and surface roughness comparisons of the resultant films for different film thicknesses. ${ }^{2}$ Here, we report a significant reduction of crystallization laser energy density required to yield a given crystalline volume by modifying the Gaussian pulse profile, while retaining the controlled evolution of hydrogen from $a$-Si:H films.

Chemical vapor deposited 300 and $500 \mathrm{~nm}$ thick $a$-Si:H on a $100 \mathrm{~nm}$ thick silicon nitride, capped Corning 1737 glass was used for the experiments. The films contain $10 \%$ atomic hydrogen, as specified by the deposition facility. A KrF Lambda Physik excimer laser (LPX 210i), operating at $248 \mathrm{~nm}$ with a $25 \mathrm{~ns}$ full width at half maximum pulse duration, was used for crystallization. The $a-\mathrm{Si}: \mathrm{H}$ films on glass were crystallized in vacuum, at a base pressure of 5 $\times 10^{-4}$ mbar, by scanning along the laser pulse. The scanning speed for the experiment was $2.5 \mathrm{~mm} \mathrm{~s}^{-1}$ with pulse repetition rate maintained at $50 \mathrm{~Hz}$. Experimental details are as similar to that used for crystallization of $a-\mathrm{Si}: \mathrm{H}$ with the Gaussian pulse profile in Ref. 2, for comparison purposes, apart from the laser pulse profile and maximum energy density used. A series of incremental energy densities of up to

${ }^{\text {a) }}$ Author to whom correspondence should be addressed; electronic mail: d.adikaari@surrey.ac.uk
$200 \mathrm{~mJ} \mathrm{~cm}^{-2}$ per pulse was used for crystallization, with $25 \mathrm{~mJ} \mathrm{~cm}^{-2}$ increments. The laser energy was measured using a Molectron EPM 1000 analyzer and a J25LP-MUV pyroelectric detector. Due to the pulse-to-pulse variation of the excimer laser energy, it is estimated that the measured energy densities have an error of up to $10 \% .^{10}$

The laser pulse incident on the silicon film was imaged using a set of image capture apparatus involving a camera. The image yields the shape of the laser pulse, normalized to the peak energy density of the pulse. For this work, a 4 $\times 8 \mathrm{~mm}^{2}$ laser pulse was used for crystallization. The laser energy is constant along the short axis of the pulse profile, whereas the long axis was an asymmetric peak profile; with a steeper trailing edge compared with the leading edge. The pulse profile was arrived at mathematically, by curve fitting using 20 data points along the long axis of the captured image of the pulse. The same procedure was followed to model the Gaussian pulse profile used in Ref. 2, which is of similar width but $10 \mathrm{~mm}$ long. Since the short axis energy profile is constant for both pulse shapes, the areas under the fitted curves of the long axis profile were equated and the corresponding normalized peak energy for the asymmetric pulse was calculated. Figure 1 shows the comparison of the two long axis pulse profiles, where the shaded areas under

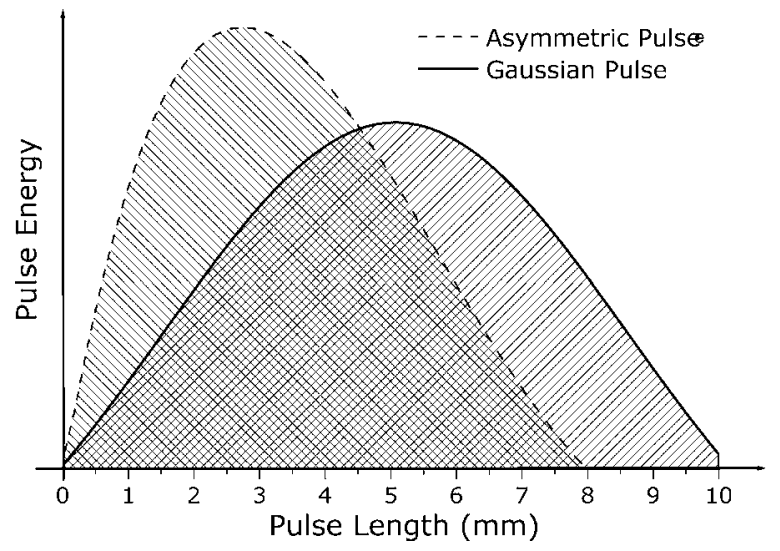

FIG. 1. Comparison of Gaussian laser pulse and asymmetric laser pulse at the same crystallization energy density. Energy is normalized to the peak energy of the Gaussian laser pulse. 


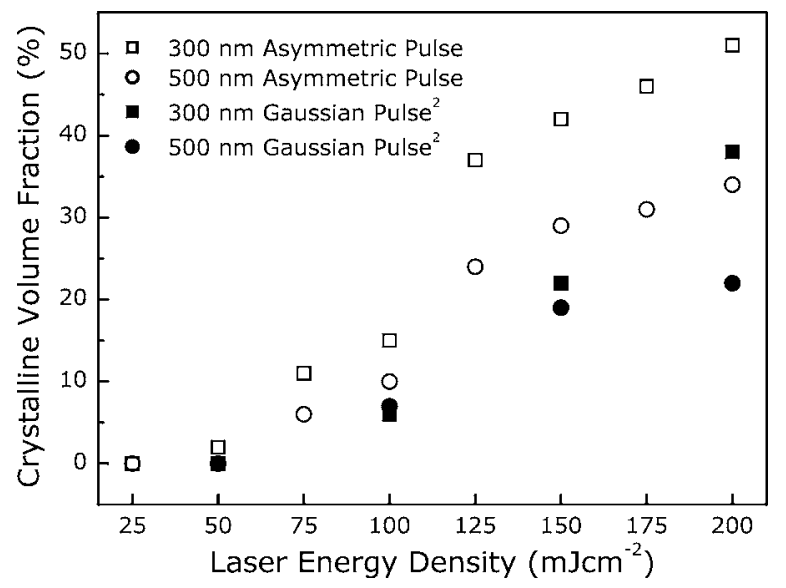

FIG. 2. Crystalline volume fractions of $300 \mathrm{~nm}$ (squares) and $500 \mathrm{~nm}$ (circles) thick excimer laser crystallized silicon calculated from Raman spectra. Solid symbols show the crystalline volume fractions of thin films crystallized with the Gaussian pulse, reproduced from Ref. 2. The open symbols represent values for laser crystallized silicon with the asymmetric pulse.

the curves are equal, and the peak energy of the asymmetric pulse is $28 \%$ higher than the peak of Gaussian pulse.

The crystalline volume fractions of the crystallized films using the asymmetric pulse were calculated from Raman spectroscopic data using the method used by Tsu et al., ${ }^{11}$ as detailed in Ref. 2. A Renishaw 2000 Ramascope was used for Raman measurements with $782 \mathrm{~nm}$ excitation. The estimated crystalline volume fractions of the resultant laser crystallized silicon films plotted against crystallization energy density are shown in Fig. 2. The error in the calculation was estimated to be $1 \%$, assuming the $782 \mathrm{~nm}$ Raman excitation laser probes to be as deep as $500 \mathrm{~nm}$. With the Gaussian pulse, evidence of crystallization is not observed for 300 or $500 \mathrm{~nm}$ thick films at $50 \mathrm{~mJ} \mathrm{~cm}$. With the asymmetric pulse, the $300 \mathrm{~nm}$ thick silicon film shows a small crystalline volume, although for the $500 \mathrm{~nm}$ films no change is observed. However, with increasing crystallization energy densities, a clear trend of higher crystalline volumes are realized for both 300 and $500 \mathrm{~nm}$ thick films, with the asymmetric pulse compared to a Gaussian pulse. The higher crystalline volume yield is consistent; however, the percentage increase of crystalline volume from Gaussian to asymmetric pulse fluctuates as the laser energy density is increased. During the scanning process, the film is imparted a higher energy density over a small area at the peak, which decides the maximum melt depth of the silicon film. It is argued that the higher peak energy of the asymmetric pulse is the fundamental reason for the higher crystalline volume yield.

Higher peak energy is realized by adjusting two parameters of the laser pulse; the length of the laser pulse and the off setting of the peak. Off setting of the peak allows us to attain a gradual increase of laser energy on the leading edge. The gradual increase of energy is expected to help control the evolution of hydrogen during crystallization, which is the main advantage of using a Gaussian pulse profile. ${ }^{8}$ Controlled evolution of hydrogen results in smoother film surfaces after crystallization, which is of importance for thin film transistor applications. The surface roughness of the laser crystallized silicon with the asymmetric pulse was estimated by atomic force microscopy over a $25 \mu \mathrm{m}^{2}$ area using a Digital Instruments Dimension 3100 scanning probe microDownloaded 31 Mar 2009 to 131.227.178.132. Redistribution subje

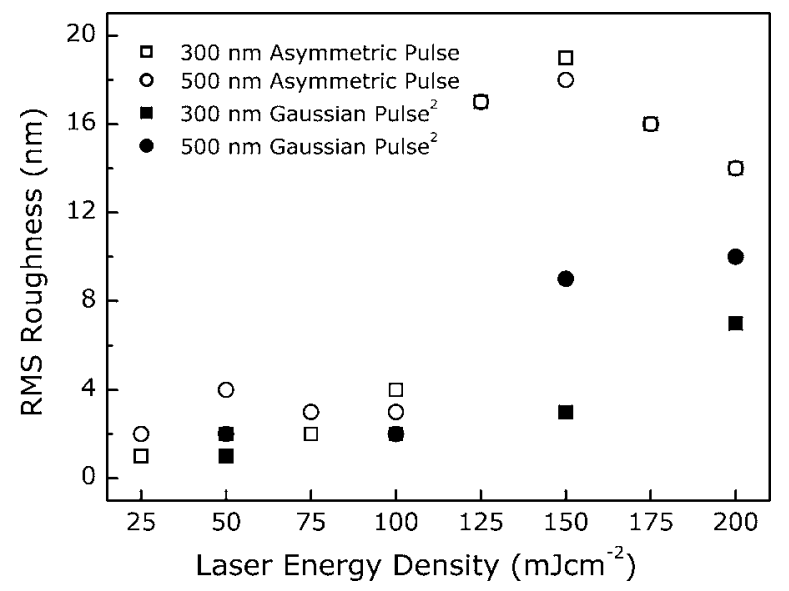

FIG. 3. rms roughness of $300 \mathrm{~nm}$ (squares) and $500 \mathrm{~nm}$ (circles) thick excimer laser crystallized silicon obtained from atomic force microscopy over a $25 \mu \mathrm{m}^{2}$ area. Solid symbols show the roughness of thin films crystallized with the Gaussian laser pulse, reproduced from Ref. 2. The open symbols represent values for laser crystallized silicon with the asymmetric laser pulse.

scope in atomic force microscopy mode. Figure 3 shows the rms roughness of the laser crystallized silicon, for both Gaussian and asymmetric pulses. Up to a laser energy density of $100 \mathrm{~mJ} \mathrm{~cm}{ }^{-2}$, rms roughness of laser crystallized silicon using both shapes of the laser pulse is $\sim 2 \mathrm{~nm}$, which is similar to the surface roughness of $a-\mathrm{Si}: \mathrm{H}$ of both 300 and $500 \mathrm{~nm}$ thicknesses. However, above $100 \mathrm{~mJ} \mathrm{~cm}^{-2}$, the surface roughness of films crystallized with the asymmetric pulse increase rapidly compared to films crystallized with the Gaussian pulse. Both thicknesses show an increased surface roughness at higher energy densities. However, the roughness values for 300 and $500 \mathrm{~nm}$ thick films crystallized with the asymmetric pulse are similar, indicating similar crystallization dynamics for both thicknesses of the films. Films crystallized with a Gaussian pulse do not show this similarity, as is evident from the reproduced roughness data from Ref. 2.

The increased surface roughness can be explained using the higher peak energy realized with the asymmetric function. The $a-\mathrm{Si}: \mathrm{H}$ films are expected to contain a couple of nanometers thick native oxide layer, ${ }^{12}$ which is expected to be transparent to $248 \mathrm{~nm}$ excimer laser $(5 \mathrm{eV})$. Therefore, the underlying $a$-Si: $\mathrm{H}$ layer is expected to melt following the energy profile of the pulse. During crystallization, at and close to the highest energy point, the films must be subjected to some level of ablation, removing material out including the native oxide which can lead to increased surface roughness. Further, it has been suggested by McCulloch and Brotherton $^{8}$ that the surface roughness from the previous shots would act as a diffraction grating, creating diffracted waves parallel to the surface. These parallel waves are believed to be interfering with the incident wave. Where a sufficiently high maximum occurs, surface modifications take place, such as local melting and surface roughness effects. Therefore, successive laser pulses would further enhance the roughness, initiated by roughness created by initial pulse due to ablation.

The fluctuation in surface roughness values for the 300 and $500 \mathrm{~nm}$ films when crystallized with a Gaussian pulse can also be explained using ablation at the peak energy. Since pulse-to-pulse variation is about $10 \%$ for the excimer to AIP license or copyright; see http://apl.aip.org/apl/copyright.jsp 
laser, ${ }^{10}$ roughness variation from ablation can be random at lower peak energies experienced using a Gaussian pulse. When peak energy is high, adequate energy can be expected to be always imparted at the peak, after a threshold energy density. Increased surface roughness may be undesirable for thin film transistor applications. However, the rough profile of the surface may be a useful by-product of the process for photovoltaics since it can be utilized as a light scattering mechanism. Also, the increased roughness can be advantageous for electron field emission applications, reducing the emission threshold due to geometric field enhancement. ${ }^{13}$

In conclusion, excimer laser crystallized silicon thin films with higher crystalline volumes are achieved with an asymmetric profile laser pulse, compared with the conventional Gaussian profile pulse, at a given laser energy density. The resultant crystalline films show increased surface roughness believed to be arising from ablation at the increased peak energy during crystallization. An optimum shape of the pulse must be possible to achieve by continuously varying the two parameters of the pulse profile: length and peak offset. The crystallization regime promises tunability for different roughness requirements depending on the application, while reducing the thermal budget.
The authors thank J. M. Shannon and Phillips Research Laboratories, Redhill, Surrey, UK, for the supply of $a-\mathrm{Si}: \mathrm{H}$ samples. This work is supported by the Portfolio Partnership Award from EPSRC.

${ }^{1}$ S. D. Brotherton, Semicond. Sci. Technol. 10, 721 (1995).

${ }^{2}$ A. A. D. T. Adikaari and S. R. P. Silva, J. Appl. Phys. 97, 114305 (2005).

${ }^{3}$ J. S. Im, H. J. Kim, and M. O. Thompson, Appl. Phys. Lett. 63, 1969 (1993).

${ }^{4}$ P. Mei, J. B. Boyce, M. Hack, R. A. Lujan, R. I. Johnson, G. B. Anderson, D. K. Fork and S. E. Ready, Appl. Phys. Lett. 64, 1132 (1994).

${ }^{5}$ G. Ivlev, E. Gatskevich, V. Chab, J. Stuchlik, V. Vorlicek, and J. Kocka, Appl. Phys. Lett. 75, 498 (1999).

${ }^{6}$ N. H. Nickel and K. Brendel, Appl. Phys. Lett. 82, 3029 (2003).

${ }^{7}$ A. A. D. T. Adikaari, J. D. Carey, V. Stolojan, J. L. Keddie, and S. R. P. Silva, Nanotechnology 17, 5412 (2006).

${ }^{8}$ D. J. McCulloch and S. D. Brotherton, Appl. Phys. Lett. 66, 2060 (1995). ${ }^{9}$ S. D. Brotherton, D. J. McCulloch, and M. J. Edwards, Solid State Phenom. 37-38, 299 (1994).

${ }^{10}$ A. T. Voustas, Appl. Surf. Sci. 208-209, 250 (2003).

${ }^{11}$ R. Tsu, J. Gonzalez-Hernandez, S. S. Chao, S. C. Lee, and K. Tanaka, Appl. Phys. Lett. 40, 534 (1982).

${ }^{12}$ R. A. Street, Hydrogenated Amorphous Silicon (Cambridge University Press, Cambridge, 1991), Vol. 1, p. 335.

${ }^{13}$ Y. F. Tang, S. R. P. Silva, B. O. Boskovic, J. M. Shannon, and M. J. Rose, Appl. Phys. Lett. 80, 4154 (2002). 